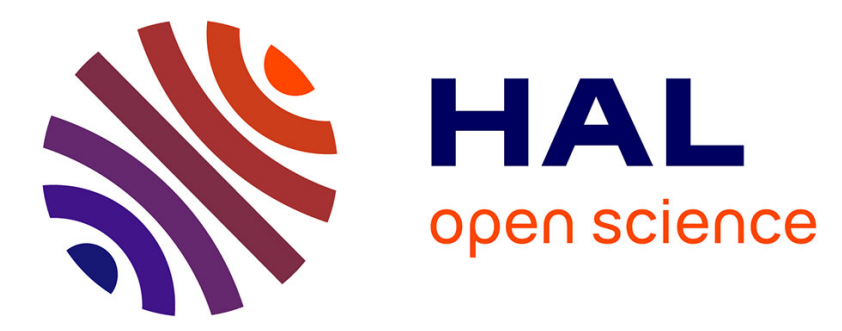

\title{
ULTIMA: Magnetic field dependence of the calibration factor
}

Johannes Elbs, Clemens Winkelmann, Yuriy M. Bunkov, Eddy Collin, Henri Godfrin

\section{- To cite this version:}

Johannes Elbs, Clemens Winkelmann, Yuriy M. Bunkov, Eddy Collin, Henri Godfrin. ULTIMA: Magnetic field dependence of the calibration factor. Journal of Low Temperature Physics, 2008, 151, pp.860. 10.2007/s10909-008-9756-z . hal-00921388

\section{HAL Id: hal-00921388 https://hal.science/hal-00921388}

Submitted on 20 Dec 2013

HAL is a multi-disciplinary open access archive for the deposit and dissemination of scientific research documents, whether they are published or not. The documents may come from teaching and research institutions in France or abroad, or from public or private research centers.
L'archive ouverte pluridisciplinaire HAL, est destinée au dépôt et à la diffusion de documents scientifiques de niveau recherche, publiés ou non, émanant des établissements d'enseignement et de recherche français ou étrangers, des laboratoires publics ou privés. 


\author{
J. Elbs · C. Winkelmann · Yu. M. Bunkov · \\ E. Collin · H. Godfrin
}

\title{
ULTIMA: Magnetic field dependence of the calibration factor
}

Keywords superfluid ${ }^{3} \mathrm{He}$, bolometry, magnetic field dependence

Abstract ULTIMA is a project which proposes to use superfluid ${ }^{3} \mathrm{He}$ as a sensitive medium for direct dark matter search. In this paper we report on new, detailed calibrations of our bolometric cells as a function of the magnetic field. An influence on the order of $20 \%$ is observed for magnetic fields up to $330 \mathrm{mT}$. Simultaneous measurements of neutron capture and heater events, releasing both a well defined energy, show that the effect is similar for both, and that it is possible to maintain a good calibration by an appropriate correction.

\section{INTRODUCTION}

The basic building block of our bolometers is a closed cell with a small orifice, immersed into superfluid ${ }^{3} \mathrm{He}$ at very low temperatures. At temperatures $T \approx 0.15 T_{C}$, where $T_{C}$ is the superfluid transition temperature, the heat capacity is small enough that if one measures the temperature inside the cell, this can be used for detecting elementary particle events ${ }^{1,2}$.

A substantial advantage of this type of bolometers is the possibility to calibrate it by direct heating of the quasiparticles by a mechanical moving object. For the purpose of both, thermometry and heating, we have used Vibrating Wire Resonators (VWR). VWR thermometers at ultra low temperatures are well studied objects $^{3,4}$. For the calibrated heat pulses we have used a second VWR also located in the cell ${ }^{5}$. The direct calibration procedure makes this type of bolometers a very special particle detector, as it does not rely on a relative or "theoretical" calibration.

Institut Néel, CNRS/UJF,

25, av. des Martyrs, BP 166, 38042 Grenoble cedex 9, France

Tel.: +33476881252

Fax: +33476875060

E-mail: yuriy.bunkov@grenoble.cnrs.fr 
Until now, the magnetic field dependence of this calibration was considered to be negligible. New measurements show that with fields up to $330 \mathrm{mT}$, the calibration factor changes by as much as $20 \%$. Even if under usual working conditions the magnetic field is limited to about $120 \mathrm{mT}$, for precise calibrations, magnetic field corrections thus have to be applied.

\section{BOLOMETRIC CALIBRATION BY HEATER PULSES}

The bolometrical cells are $0.13 \mathrm{~cm}^{3}$ copper boxes filled with superfluid ${ }^{3} \mathrm{He}-\mathrm{B}$ and linked to an outer ${ }^{3} \mathrm{He}-\mathrm{B}$ reservoir by a small orifice. The temperature inside the cell is monitored by a Vibrating Wire Resonator (VWR). The damping of the VWR is dominated by the interaction with the quasiparticle gas, whose density exponentially depends on temperature. A frequency sweep over the resonance thus delivers a Lorentzian whose FWHM $W(T)$ contains the information about the temperature. In the zero field limit, $W(T)$ writes $^{6}$

$$
W(T)=\alpha \exp \left(-\Delta / k_{B} T\right),
$$

where $\Delta$ is the superfluid gap at zero temperature and zero field, and $\alpha$ a prefactor which depends on the geometry of the VWR and pressure dependent properties of the liquid. For to obtain this equation, the influence of quasiparticles incoming from all different directions $\hat{k}$ has to be integrated. At zero field, where the dispersion relation is isotropic, the integral is analytic and the above result is obtained. For finite magnetic field, the superfluid gap deforms ${ }^{7}$ and to get the corresponding relation $W(T, B)$, a correction has to be applied to the above formula. The theoretical magnetic field dependence $W(T, B)$ has not yet been determined, but measurements done by the Lancaster group ${ }^{8}$ give experimental evidence that for non zero field, $W$ indeed increases.

After a heating event caused for example by a cosmic particle, the temperature inside the cell will suddenly rise, and then go back to its initial temperature by thermalisation via the hole. A method used to simulate such an event is to introduce by a second VWR a well known energy by mechanical friction ${ }^{2,9}$ (see fig. 1) For small enough pulses, the height of the resulting peak $(H$, in $\mathrm{Hz})$ is proportional to the heat injected $(E)$, and we can define the calibration coefficient as $\sigma=H / E$. A detailed calculation shows that this calibration coefficient is proportional to the temperature derivative of $W(T), W^{\prime}(T)$ and inversely proportional to the heat capacity $(C)$ :

$$
\sigma=\frac{1}{C} \frac{d W(T)}{d T}=\frac{1}{C} W^{\prime}(T) .
$$

The heat capacity is dominated by the quasiparticle gas. As the total number of quasiparticles strongly depends on the gap, a distortion of the gap should modify this term too, so that the magnetic field dependence of the calibration coefficient is the result of both factors, $C$ and $W^{\prime}$, depending on magnetic field.

For zero field, the temperature dependence for both factors is known, and a remarkable feature of this kind of bolometer is that the exponential temperature dependence cancels, and a weak $1 / \sqrt{T}$ dependence is expected, meaning that the calibration factor does not change much over the temperature range of interest. 


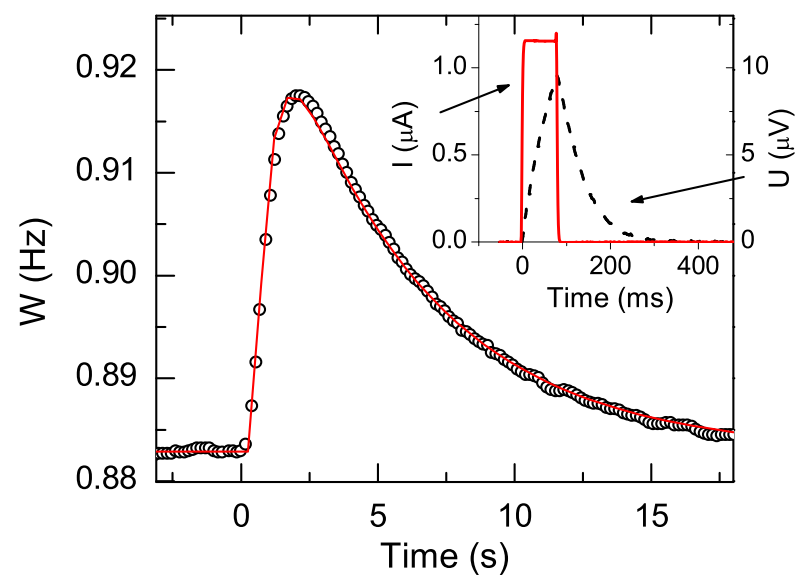

Fig. 1 (Color on-line) Example of a typical heater pulse. The heater wire is excited during a short time $(80 \mathrm{~ms})$ with a current $I$ (inset, red bold line). The VWR starts to move and induces a voltage $U$ (inset, black dashed line). The total energy $E$ injected is obtained as the integral over the product $E=\int U I d t$. At the same time, an increase of the width on the thermometer VWR can be observed (black circles). The peak shape is well understood and can be fitted (red line). After some corrections ${ }^{5}$, the peak height divided by the energy gives the calibration factor $\sigma$.

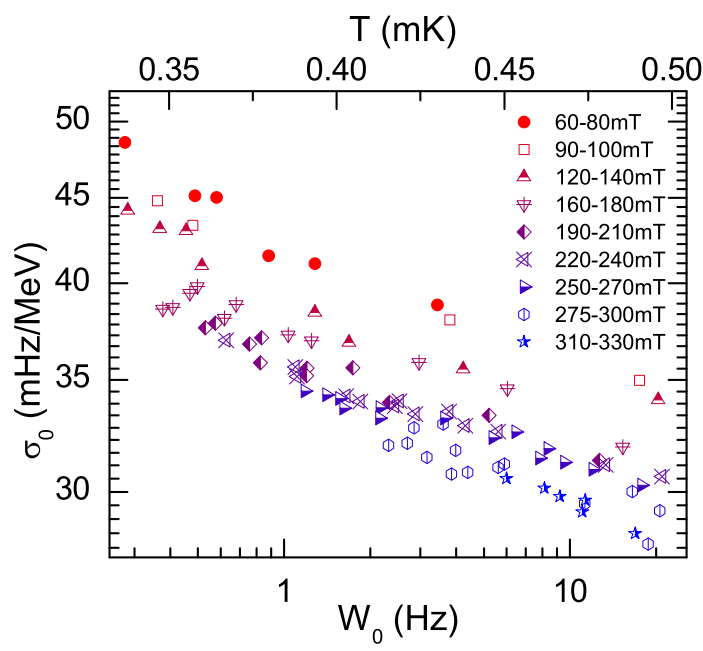

Fig. 2 (Color on-line) Calibration factor for different baseline widths and magnetic fields (29.3 bar). The exact conversion between $W(T, B)$ and the temperature is only known for $B=0 \mathrm{~T}$, so that the temperature scale on the top only has indicative character. For constant magnetic field, the expected $1 / \sqrt{T}$ dependence is found. 


\section{MAGNETIC FIELD DEPENDENCE}

The working temperatures down to $130 \mu \mathrm{K}$ are reached using a nuclear demagnetisation cryostat. We use a Lancaster style nuclear stage, so that the experimental cells are in the main coil, and the magnetic field is the final field of the demagnetisation. This in turn means that changing the field without changing the temperature is impossible. Changing the temperature at constant field can be done by heating, but due to the huge solid liquid thermal boundary resistance (Kapitza resistance), thermalisation after such heating takes to long time $(>3 \mathrm{~h})$. The most efficient method which was found was to change the magnetic field stepwise and measure at the resulting temperature. Fig. 2 presents the results from calibration pulses, all taken during one demagnetisation run. The data are taken at different "baseline widths", i.e. the equilibrium VWR width $W_{0}$ observed between events. Each point corresponds to a total of 25 heater pulses with different heating powers. The calibration factor $\sigma$ is then taken as the slope in the peak height-energy plot. It can be clearly seen that the magnetic field has a systematic influence. The magnetic field dependence of $\sigma$ for constant temperature would be interesting for a quantitative study of this phenomena, but unfortunately no independent thermometry exists, and the field correction for the VWR width is not known. In fig. $3, \sigma^{-1}$ as function of magnetic field is plotted for three different baseline widths. This graph as well as similar graphs for other $W_{0}$ show linear behavior, with almost the same slope for the different temperatures. The total of experimental results can thus be described well by

$$
\sigma(W, B)=\frac{\beta}{\sqrt{T(W)}} \cdot \frac{1}{1+\gamma B},
$$

where $\beta$ is a constant which depends on superfluid ${ }^{3} \mathrm{He}$ parameters and the VWR geometry, $T(W)$ is the temperature as obtained using the zero field formula (eq. 1), and $\gamma$ a phenomenological constant.

\section{DISCUSSION}

The presented results were taken at $29.3 \mathrm{bar}$, because at higher pressures, the superfluid transition temperature $T_{C}$ is higher and a higher field range can be measured. Measurements at lower pressures qualitatively show the same behavior.

The linear dependence in $B$ for constant baseline width is at first very surprising, especially because one expects for symmetry reasons a quadratic correction in field for both, the specific heat and the VWR width. Preliminary numerical simulations however show that under certain conditions, quadratic field corrections for constant temperatures can lead to a linear correction of calibration factor for constant baseline width. More detailed simulations are still a work in progress.

A second method which can be used for calibration is to use a neutron source for the production of nuclear capture reactions. This reaction releases a well defined energy of about $764 \mathrm{keV}$, of which about $652 \mathrm{keV}$ are transfered to heat. Using this second method and applying at the same time heater pulses demonstrates that the influence of the magnetic field is not limited to heater pulses. This 


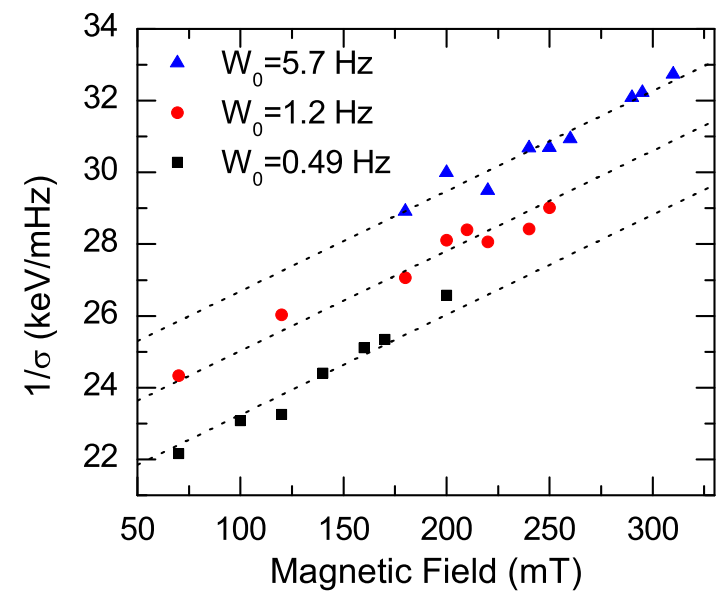

Fig. 3 (Color on-line) $1 / \sigma$ for three different, almost constant baseline widths $W_{0}$. Similar graphs at different $W_{0}$ indicate that for the whole measured range 0.2 to $20 \mathrm{~Hz}, 1 / \sigma$ is linear with magnetic field strength: $\sigma\left(W_{0}=c s t\right) \propto 1 /(1+\gamma B)$. The precision is not sufficient to conclude whether or not $\gamma$ varies with $W_{0}$ but in any case, the dependence is rather weak.

is a very important observation for the ULTIMA project, as it shows that the influence of the magnetic field introduces an additional correction factor which needs to be considered, but that it does not prevent achieving a proper energy calibration.

To conclude, we observed a magnetic field dependence of the calibration factor. While a complete numerical understanding is still missing, a phenomenological characterisation is possible. Accounting for this effect opens the way for a future dark matter detector with an accurate energy resolution.

\section{ACKNOWLEDGMENTS}

This work was done in the framework of the ULTIMA project of the "Agence Nationale de la Recherche", France. We are thankful to the ESF Program COSLAB for many stimulating discussions during workshops and conferences.

\section{References}

1. D. I. Bradley, Yu. M. Bunkov, D. J. Cousins, M. P. Enrico, S. N. Fisher, M. R. Follows, A. M. Guénault, W. M. Hayes, G. R. Pickett and T. Sloan, Phys. Rev. Lett. 751887 (1995)

2. C. Bäuerle, Yu. M. Bunkov, S. N. Fisher and H. Godfrin, Phys. Rev. B 57, 14381 (1998).

3. A. M. Guénault, V. Keith, C. J. Kennedy S. G. Mussett, and G. R. Pickett, J. Low Temp. Phys. 62, 511 (1986)

4. C. B. Winkelmann, E. Collin, Yu. M. Bunkov and H. Godfrin, J. Low Temp. Phys. 135, 3 (2004) 
5. C. B. Winkelmann, J. Elbs, E. Collin, Yu. M. Bunkov and H. Godfrin, Nucl. Instr. and Meth. A 574, 264 (2007)

6. S. N. Fisher, G. R. Pickett and R. J. Watts-Tobin, J. Low Temp. Phys. 83, 225 (1991).

7. L. Tewordt N. and Schopohl, J. Low Temp. Phys. 37, 421 (1979)

8. D. I. Bradley, S. N. Fisher, A. M. Guénault, R. P. Haley, H. Martin, G. R. Pickett, J. Low Temp. Phys. 138, 583 (2005)

9. S. N. Fisher, A. M. Guénault, C. J. Kennedy and G. R. Pickett, Phys. Rev. Lett. 69, 1073 (1992). 